\title{
ASSESSMENT OF HEMATOLOGIC INDICES AND THEIR CORRELATION TO HEMOGLOBIN A1C AMONG BOSNIAN CHILDREN WITH TYPE 1 DIABETES MELLITUS AND THEIR HEALTHY PEERS
}

\author{
PROCENA HEMATOLOŠKIH PARAMETARA I NJIHOVE KORELACIJE SA HEMOGLOBINOM \\ A1C KOD BOSANSKE DECE SA TIPOM 1 DIJABETES MELITUSA I ZDRAVIH VRŠNJAKA
}

\author{
Suzana Tihić Kapidžić ${ }^{1}$, Adlija Čauševićé, Jasmina Fočo Solak , Maja Malenica², \\ Tanja Dujić2, Sniježana Hasanbegović3 ${ }^{3}$ Nermina Babić ${ }^{4}$, Ermin Begović ${ }^{1}$ \\ ${ }^{1}$ Department for Clinical Biochemistry and Immunology, Clinical Centre University of Sarajevo, \\ Bosnia and Herzegovina \\ ${ }^{2}$ Department of Biochemistry and Clinical Analysis, Faculty of Pharmacy, University of Sarajevo, \\ Bosnia and Herzegovina \\ ${ }^{3}$ Pediatric Clinic, Clinical Centre University of Sarajevo, Bosnia and Herzegovina \\ ${ }^{4}$ Department of Human Physiology, Medical Faculty, University of Sarajevo, Bosnia and Herzegovina
}

\section{Summary}

Background: Altered levels of many hematological parameters have been directly associated with diabetes in adults, while studies on children with type 1 diabetes mellitus are lacking. The aim of this study was to determine hematological indices in diabetic Bosnian children in comparison to healthy controls as well as to correlate their levels to blood glucose and hemoglobin A1c.

Methods: 100 healthy and 100 children with type 1 diabetes mellitus (age 1-18) were included in this study. Complete blood count, hemoglobin A1c, and glucose were tested. Results were analysed by IBM SPSS Statistics version 23.

Results: Significant differences $(p<0.05)$ between healthy and diabetic children were found in relation to $\mathrm{HbA} 1 \mathrm{c}$, glucose, mean platelet volume, the number of white blood cells and erythrocytes, hematocrit, hemoglobin and $\mathrm{MCH}$ values. No gender differences or significant age differences were seen for hemoglobin, hematocrit, and $\mathrm{MCV}$, while platelets, MPV, and $\mathrm{MCH}$ differed by age only in healthy children. When diabetic children were classified according to $\mathrm{HbA} 1 \mathrm{c}$ levels, significant differences were seen for ery-

Address for correspondence:

Suzana Tihić Kapidžić

25 Bolnička Street, 71000 Sarajevo, Bosnia and Herzegovina

Fax: + 38733297501

Phone: +38761222197

e-mail: suzanakapidzic@yahoo.com

\section{Kratak sadržaj}

Uvod: Brojne studije ukazuju na promene hematološkog statusa kod odrasle populacije sa dijabetesom, dok su istraživanja na deci sa tipom 1 dijabetes melitusa nedovoljna. Cilj ovog istraživanja je bio utvrditi hematološki status kod dijabetične bosanske dece i uporediti ga sa zdravom kontrolnom grupom te analizirati vezu hematoloških parametara sa koncentracijama glukoze i hemoglobina A1c u serumu.

Metode: U istraživanje je uključeno 100 zdrave i 100 dece sa tipom 1 dijabetes melitusa, starosti od 1 do 18 godina. Kompletna krvna slika, hemoglobin A1c i glukoza su testirani kod sve dece. Analiza podataka je urađena pomoću programa IBM SPSS Statistics, verzija 23.

Rezultati: Značajne razlike $(p<0,05)$ između zdrave i dijabetične dece su uočene u vrednostima HbA1c, glukoze, MPV, broju leukocita i eritrocita, hematokrita, hemoglobina i $\mathrm{MCH}$. Polne razlike u vrednostima ispitivanih parametara nisu nađene. Starosne razlike su bile značajne za vrednosti hemoglobina, hematokrita i MCV. Broj trombocita, MPV i $\mathrm{MCH}$ su pokazali starosne razlike samo kod zdrave dece. Podela dijabetične dece prema vrednostima HbA1c je

List of abbreviations: $\mathrm{BMI}$, body mass index; $\mathrm{CBC}$, complete blood count; HbA1c, hemoglobin A1c; $\mathrm{HCT}$, hematocrit; $\mathrm{HGB}$, hemoglobin; IQR, interquartile range; $M C H$, mean corpuscular hemoglobin; $\mathrm{MCHC}$, mean corpuscular hemoglobin concentration; MCV, mean corpuscular volume; $M D$, medical doctor; MPV, mean platelet volume; PLT, platelets; RBC, red blood cells; RDW, red blood cell distribution width; rpm, revolutions per minute; T1DM, type 1 diabetes mellitus; T2DM, type 2 diabetes mellitus; WBC, white blood cells. 
throcyte count and hematocrit value $(p=0.013$ and 0.019 , respectively). The number of erythrocytes and white blood cells correlated significantly with $\mathrm{HbA} 1 \mathrm{c}(\mathrm{p}=0.037$ and 0.027 , respectively).

Conclusions: Lower levels of erythrocytes, hematocrit, and hemoglobin in diabetic compared to healthy children indicate possible development of anemia, while higher MCV, $\mathrm{MCH}$, and MPV values indicate an alteration in erythrocyte morphology. Hematological indices could be a useful inexpensive tool in the diagnosis and follow up of type 1 diabetes in children.

Keywords: hematological indices, hemoglobin A1c, type 1 diabetes mellitus, pediatric population

\section{Introduction}

Type 1 diabetes mellitus (T1DM) is one of the most common endocrinological disorders in children and adolescents $(1,2)$. It is caused by various environmental factors interacting with an underlying genetic predisposition resulting in autoimmune destruction of pancreatic beta cells $(3,4)$. The onset of type 1 diabetes mellitus occurs early in life and children affected are at great risk of developing microvascular and macrovascular complications and cardiovascular disease later as a complication of diabetes. Hyperglycemia, dyslipidemia, inflammation, and stress are factors that increase the risk of vascular complications in patients with $\operatorname{T1DM}(2,8)$. Those long-term complications are the leading cause of premature mortality in this group of patients (5-7). T1DM complications that develop in children and adolescents depend on disease duration, metabolic control level, environmental and genetic factors.

T1DM and its complications represent a heavy financial burden on diabetic patients, their families, and society. This is especially true in developing countries. Hematological indices from complete blood counts may be used to overcome these challenges as good indicators and independent predictors for various microvascular and macrovascular complications related to endothelial dysfunction and inflammation. Altered level of many hematological parameters such as red blood cells (RBCs), white blood cells (WBC), and the platelet function and morphology have been observed and shown to be directly associated with diabetes $(1-4,12,13,15-18,25-28)$ and metabolic syndrome in the adult population, indicating their close relationship with different components of metabolic syndrome, including insulin resistance (913). These studies demonstrated a significant difference in red blood cell distribution width as well as platelet volume between type 2 diabetic patients and controls (both increased in diabetic patients) (9-11). Differences also manifested at the level of total white blood cells, absolute lymphocyte count, absolute neutrophil count when compared to controls (all three increased significantly in diabetic patients) (10).

Biadgio et al. (10), found that among platelet indices, mean platelet volume, and platelet distribu- pokazala da postoje značajne razlike samo u broju eritrocita $i$ vrednosti hematokrita $(p=0,013$ i 0,019, respektivno). Broj eritrocita i leukocita je pokazao statistički značajne pozitivne korelacije sa $\mathrm{HbA} 1 \mathrm{c}(\mathrm{p}=0,037$ i 0,027 , respektivno).

Zaključak: Niže vrednosti eritrocita, hematokrita i hemoglobina kod dijabetične $u$ odnosu na zdravu decu indiciraju potencijalni razvoj anemije, dok više vrednosti $\mathrm{MCV}, \mathrm{MCH}$ i MPV ukazuju na morfološke promene eritrocita. Hematološki parametri mogu biti jeftini $i$ korisni pokazatelji u dijagnozi i praćenju dece sa tipom 1 dijabetes melitusa.

Ključne reči: hematološki parametri, hemoglobin A1c, dijabetes melitus tip 1, deca

tion width were significantly increased in diabetic patients. In their study, some of the parameters of complete blood count (CBC) were found to be useful tools in following type 2 diabetes and its complications.

Milosevic et al. (11) reported independent associations of hematological parameters with insulin resistance and glycemic control. This association was also reported by Uko et al. (14) in type 1 diabetic patients, where changes in packed cell volume, platelet, and total white cell count as well as anemia, thrombocytosis and leukocytosis were reported.

Studies related to the issue of diagnostic significance of different hematological markers in children diabetic population are lacking worldwide due to unrecognized steep rise in the incidence of childhood diabetes affecting all countries in the world, including Bosnia and Herzegovina. Therefore, the aim of this study was to determine hematological indices (RBC count, hemoglobin, hematocrit, $\mathrm{MCV}, \mathrm{MCH}, \mathrm{MCHC}$, RDW, PLT count, platelet morphology, platelet activation indicator MPV) in Bosnian children with T1DM and compare them to healthy controls of the same age as well as to correlate their levels to blood glucose, HbA1c levels, and anthropometric measurements. Of particular interest was to compare the sex and age differences in the examined parameters. To our knowledge, this is the first study of this kind in Bosnia and Herzegovina.

\section{Materials and Methods}

\section{Patient population and study design}

Two hundred children aged between 1-18 years were included in this study. The whole study group $(\mathrm{N}=200)$ consisted of 100 (50\%) healthy children and 100 (50\%) children with type 1 diabetes mellitus treated with insulin at the Department of Paediatrics, University Clinical Centre Sarajevo. The diabetic group was at the later phase divided into three groups according to HbA1c levels.

The control group was age- and sex-matched to the study group. The mean age of children enrolled 
in the study was $10.36 \pm 3.85$ years. In a group with T1DM, 13 (13\%) children were 1-6 years old, 58 (58\%) were 7-12 years old, and 29 (29\%) 13-18 years old. In the healthy control group, 15 (15\%) children were 1-6 years old, 58 (58\%) were 7-12 years old, and 27 (27\%) 13-18 years old. The male to female ratio in the control group was 1.2, and 1.0 in children with T1DM. The group of children with T1DM consisted of 50 (50\%) male and 50 (50\%) female subjects, while the control group consisted of 55 (55\%) male and 45 (45\%) female.

Written informed consent was obtained from all participants recruited into this study (controls and diabetic patients). The study was approved by the Ethical Committee of the University Clinical Centre Sarajevo and was carried out in compliance with the Helsinki Declaration.

After obtaining detailed histories for all the children involved in the study, they were physically examined by a medical doctor (MD), and data were recorded on forms prepared for this study. Collected data included age (year), sex, height $(\mathrm{cm})$, weight $(\mathrm{kg})$, body mass index $(\mathrm{BMI})\left(\mathrm{kg} / \mathrm{m}^{2}\right)$, family history of chronic diseases, and the laboratory findings. Patients with a history of accompanying chronic disease, current acute infection, alcohol abuse, and atherosclerotic disease or with severe hypoglycemia or diabetic ketoacidosis were excluded from the study.

\section{Laboratory tests}

The following laboratory tests were performed on healthy and children with T1DM: white blood cell count $\left(10^{12} / \mathrm{L}\right)$, red blood cell count $\left(10^{12} / \mathrm{L}\right)$, hemoglobin $(\mathrm{g} / \mathrm{L})$, hematocrit $(\%), M C V(\mathrm{fL}), \mathrm{MCH}$ $(\mathrm{pg}), \operatorname{MCHC}(\mathrm{g} / \mathrm{L}), \mathrm{RDW}(\% \mathrm{CV})$, platelet count $\left(10^{9} / \mathrm{L}\right), \mathrm{MPV}(\mathrm{fL}), \mathrm{HbA} 1 \mathrm{c}(\%)$, glucose $(\mathrm{mmol} / \mathrm{L})$. Blood samples were taken from the brachial vein after overnight fasting and were processed within 2 hours after collection, according to the standard operating procedure of our institution. Glucose and HbA1c levels were acquired from blood samples that were centrifuged in gel tubes (3000 rpm for 5 minutes). Red blood cell and platelet morphology parameters were extracted from routinely performed complete blood count results. The blood counts were performed on Cell-Dyn Sapphire (Abbott Diagnostic, USA). Glucose levels were determined with Architect ci8200 (Abbott Diagnostics, USA), and $\mathrm{HbA} 1 \mathrm{c}$ was measured using HPLC method on Adams analyzer (Arkray, USA).

\section{Statistical analysis}

Statistical evaluation of results was performed by IBM SPSS Statistics version 23 (Chicago, IL, USA). Median, interquartile range (IQR), ratio, and frequency values were used for the descriptive statistics of the data. Data distribution was tested using the Kolmo-
gorov-Smirnov test. The parameters were compared within two groups using the Mann-Whitney $U$ test. Spearman's rho correlation analysis was used to assess the relationships between the parameters. The Kruskal-Wallis test was used for the comparison of multiple groups. P-value $<0.05$ was considered statistically significant.

\section{Results}

There were no differences in age, BMI, total platelet count, MCV, MCHC, and RDW between healthy and children with T1DM (Table I). The MannWhitney $U$ test showed a significant difference between the healthy control group and children with T1DM in HbA1c, glucose, MPV, number of white blood cells, number of erythrocytes, hematocrit and hemoglobin values, with $\mathrm{p}<0.001$ for all 7 parameters (Table I). MCH also shows a significant difference between healthy and children with T1DM, with $\mathrm{p}=0.035$ (Table I).

When healthy and diabetic populations were divided according to gender, statistically significant gender differences were observed only at the level erythrocyte count, hemoglobin, and MCV $(p=0.007$, 0.048 , and 0.012 ) in the group of diabetic patients (Table II). Healthy controls showed no gender differences in any of the examined parameters (Table II).

In the analysis of the study populations according to their age, hemoglobin, hematocrit, and MCV showed statistically significant differences between compared age groups, both in diabetic children and their healthy peers (Table III). MCHC and RDW were significantly different within compared age groups in diabetic children $(p=0.02$ and $p=0.007$, respectively), while platelets, MPV, and MCH showed statistically significant age differences in healthy children ( $p=0.06, p=0.06$, and $p=0.01$, respectively) (Table III). There were no other statistically significant age differences within the study groups except for those expected, such as age and BMI (Table III).

In the next phase, the effects of glycaemia control were tested in the population of diabetic children. After baseline evaluation, diabetic patients were divided into three groups according to their $\mathrm{HbA} 1 \mathrm{c}$ levels: Group A consisted of patients with $\mathrm{HbA} 1 \mathrm{c}$ levels $<7 \%$, group $\mathrm{B}$ consisted of patients with $\mathrm{HbA} 1 \mathrm{c}$ levels between 7.1-8.4 and group $\mathrm{C}$ with $\mathrm{HbA} 1 \mathrm{c}$ 8.5\% (Table IV). The latest blood A1C targets for children and adolescents with type 1 diabetes, according to the American Diabetic Association 2019 criteria (15) is $7.5 \%$. A lower goal $(<7.0 \%)$ is reasonable if it can be achieved without excessive hypoglycemia.

All of the healthy children in the control group had HbA1c values $<6 \%$ (Table IV). According to $\mathrm{HbA} 1 \mathrm{c}$ values, $26 \%$ of children had an excellent level of disease control, $44 \%$ of children showed a some- 
Table I Anthropometric, clinical and hematological characteristics of children with type 1 diabetes mellitus and healthy controls.

\begin{tabular}{|c|c|c|c|}
\hline Parameter & $\begin{array}{l}\text { Healthy Controls } \\
\qquad(N=100)\end{array}$ & $\begin{array}{c}\text { Type } 1 \text { diabetes } \\
(N=100)\end{array}$ & $\mathrm{p}$ \\
\hline Age (years) & $9.0(8.0-13.0)$ & $10.5(8.0-14.0)$ & 0.071 \\
\hline $\mathrm{BMI}\left(\mathrm{kg} / \mathrm{m}^{2}\right)$ & $18.5(15.8-21.3)$ & $18.4(16.3-20.2)$ & 0.920 \\
\hline HbA1c (\%) & $5.1(4.9-5.2)$ & $7.9(7.0-8.7)$ & $<0.001$ \\
\hline Platelets $\left(10^{9} / \mathrm{L}\right)$ & $286.5(253.5-330.8)$ & $274.5(235.3-326.8)$ & 0.141 \\
\hline MPV (fL) & $6.3(5.6-6.8)$ & $7.5(6.5-9.3)$ & $<0.001$ \\
\hline Glucose $(\mathrm{mmol} / \mathrm{L})$ & $4.7(4.4-5.0)$ & $13.5(9.3-18.3)$ & $<0.001$ \\
\hline $\operatorname{RBC}\left(10^{12} / \mathrm{L}\right)$ & $5.2(4.9-5.4)$ & $4.9(4.6-5.1)$ & $<0.001$ \\
\hline Hemoglobin $(\mathrm{g} / \mathrm{L})$ & $143.0(138.0-148.0)$ & $137.0(129.3-143.8)$ & $<0.001$ \\
\hline Hematocrit (\%) & $43.3(41.4-45.1)$ & $40.7(39.1-43.7)$ & $<0.001$ \\
\hline $\mathrm{MCV}(\mathrm{fL})$ & $82.9(81.3-85.7)$ & $83.4(80.6-88.3)$ & 0.476 \\
\hline $\mathrm{MCH}(\mathrm{pg})$ & $27.5(26.8-28.5)$ & $28.1(27.0-29.2)$ & 0.035 \\
\hline $\mathrm{MCHC}(\mathrm{g} / \mathrm{L})$ & $330.0(327.0-336.0)$ & $332.5(320.0-348.0)$ & 0.514 \\
\hline RDW (\%CV) & $11.8(11.4-12.1)$ & $11.6(10.6-12.4)$ & 0.088 \\
\hline WBC $\left(10^{12} / L\right)$ & $7.3(5.9-8.7)$ & $6.1(4.9-7.5)$ & $<0.001$ \\
\hline
\end{tabular}

Values represent medians (lower-upper quartile), $\mathrm{p}$ - significance between healthy controls and children with type 1 diabetes. All differences were tested using the Mann-Whitney $U$ test.

$\mathrm{BMI}$, body mass index; HbA1c, hemoglobin A1c; MPV, mean platelet volume; RBC, red blood cells; MCV, mean corpuscular volume; $\mathrm{MCH}$, mean corpuscular hemoglobin; MCHC, mean corpuscular hemoglobin concentration; RDW, red blood cell distribution width; WBC, white blood cells.

Table II Gender differences in anthropometric, clinical and hematological characteristics of children with type 1 diabetes mellitus and healthy controls.

\begin{tabular}{|l|c|c|c|c|c|c|}
\hline \multirow{2}{*}{ Parameter } & \multicolumn{3}{|c|}{ Healthy Controls } & \multicolumn{3}{c|}{ Type 1 Diabetes } \\
\cline { 2 - 6 } & Male (N=55) & Female (N=45) & $p^{*}$ & Male (N=50) & Female (N=50) & $p^{* *}$ \\
\hline Age (years) & $9.0(7.0-13.0)$ & $9.0(8.0-13.0)$ & 0.564 & $10.0(7.8-12.0)$ & $12.0(9.0-15.0)$ & 0.097 \\
\hline BMI (kg/m²) & $18.6(16.4-20.4)$ & $17.4(15.4-21.6)$ & 0.716 & $16.3-19.4$ & $18.5(16.4-21.0)$ & 0.182 \\
\hline HbA1c (\%) & $5.2(4.9-5.3)$ & $5.1(4.9-5.2)$ & 0309 & $7.9(7.0-8.7)$ & $7.9(7.1-8.5)$ & 0.738 \\
\hline Platelets (109/L) & $293.0(255.0-334.0)$ & $283.0(251.5-329.0)$ & 0.554 & $272.5(229.3-315.8)$ & $276.0(239.3-339.8)$ & 0.340 \\
\hline MPV (fL) & $6.3(5.8-6.8)$ & $6.2(5.5-6.9)$ & 0.371 & $7.4(6.5-9.5)$ & $7.6(6.4-9.2)$ & 0.775 \\
\hline Glucose (mmol/L) & $4.7(4.5-5.1)$ & $4.7(4.4-4.9)$ & 0.420 & $13.1(8.7-18.9)$ & $14.4(10.0-17.3)$ & 0.863 \\
\hline RBC (1012/L) & $5.3(5.15 .4)$ & $5.1(4.9-5.4)$ & 0.190 & $5.0(4.8-5.3)$ & $4.7(4.5-5.0)$ & 0.007 \\
\hline Hemoglobin (g/L) & $144.0(138.0-149.0)$ & $143.0(138.0-147.5)$ & 0.739 & $140.5(130.8-144.3)$ & $135.5(129.0-141.0)$ & 0.048 \\
\hline Hematocrit (\%) & $43.2(41.4-45.1)$ & $43.3(41.5-45.1)$ & 0.816 & $40.8(39.3-44.1)$ & $40.4(38.9-43.1)$ & 0.377 \\
\hline MCV (fL) & $82.9(81.3-85.6)$ & $83.4(81.3-86.2)$ & 0.425 & $81.9(79.6-86.8)$ & $85.2(81.5-90.7)$ & 0.012 \\
\hline MCH (pg) & $27.5(26.8-28.4)$ & $27.7(26.9-29.0)$ & 0.427 & $27.8(26.9-28.9)$ & $28.3(27.4-29.8)$ & 0.224 \\
\hline MCHC (g/L) & $332.0(326.0-336.0)$ & $330.0(327.5-336.0)$ & 0.920 & $338.0(321.8-349.0)$ & $329.0(319.0-345.0)$ & 0.177 \\
\hline RDW (\%CV) & $11.8(11.5-12.1)$ & $11.7(11.4-12.1)$ & 0.474 & $11.7(10.5-12.3)$ & $11.5(10.7-12.4)$ & 0.823 \\
\hline WBC (1012/L) & $7.3(6.2-8.5)$ & $7.3(5.7-9.3)$ & 0.724 & $5.9(4.8-7.5)$ & $6.4(5.2-7.9)$ & 0.528 \\
\hline
\end{tabular}

Values represent medians (lower-upper quartile), $\mathrm{p}^{*}$ - significance between males and females in the group of healthy controls. $\mathrm{p}^{* *}-$ significance between males and females in children with type 1 diabetes. All differences were tested using the Mann-Whitney $U$ test.

$\mathrm{BMI}$, body mass index; HbA1c, hemoglobin A1c; MPV, mean platelet volume; RBC, red blood cells; MCV, mean corpuscular volume; $\mathrm{MCH}$, mean corpuscular hemoglobin; $\mathrm{MCHC}$, mean corpuscular hemoglobin concentration; RDW, red blood cell distribution width; WBC, white blood cells. 
Table III Age differences in anthropometric, and hematological characteristics of children with type 1 diabetes mellitus and healthy controls.

\begin{tabular}{|c|c|c|c|c|c|c|c|c|}
\hline \multirow[b]{2}{*}{ Parameter } & \multicolumn{4}{|c|}{ Healthy Controls } & \multicolumn{4}{|c|}{ Type 1 Diabetes } \\
\hline & $\begin{array}{l}1-6 \text { years } \\
(N=15)\end{array}$ & $\begin{array}{c}7-12 \text { years } \\
(N=58)\end{array}$ & $\begin{array}{c}13-18 \text { years } \\
(N=27)\end{array}$ & $p^{*}$ & $\begin{array}{c}1-6 \text { years } \\
(N=13)\end{array}$ & $\begin{array}{c}7-12 \text { years } \\
(N=58)\end{array}$ & $\begin{array}{c}13-18 \text { years } \\
(N=29)\end{array}$ & $p^{* *}$ \\
\hline Age (years) & $\begin{array}{c}4.0 \\
(3.0-5.0)\end{array}$ & $\begin{array}{c}9.0 \\
(8.0-10.3)\end{array}$ & $\begin{array}{c}14.0 \\
(13.0-16.0)\end{array}$ & $<0.001$ & $5.0(3.5-6.0)$ & $\begin{array}{c}10.0 \\
(9.0-12.0)\end{array}$ & $\begin{array}{c}16.0 \\
(14.0-17.0)\end{array}$ & $<0.001$ \\
\hline BMI $\left(\mathrm{kg} / \mathrm{m}^{2}\right)$ & $\begin{array}{c}15.2 \\
(14.8-16.4)\end{array}$ & $\begin{array}{c}18.4 \\
(15.4-20.2)\end{array}$ & $\begin{array}{c}21.4 \\
(18.6-23.2)\end{array}$ & $<0.001$ & $\begin{array}{c}18.1 \\
(15.9-19.3)\end{array}$ & $\begin{array}{c}17.2 \\
(15.8-18.6)\end{array}$ & $\begin{array}{c}21.0 \\
(19.3-23.4)\end{array}$ & $<0.001$ \\
\hline HbA1c (\%) & $5.1(4.9-5.2)$ & $5.1(4.9-5.2)$ & $\begin{array}{c}5.1 \\
(4.9-5.3)\end{array}$ & 0.928 & $8.2(7.8-8.8)$ & $\begin{array}{c}7.7 \\
(6.8-8.6)\end{array}$ & $\begin{array}{c}8.0 \\
(7.3-8.9)\end{array}$ & 0.656 \\
\hline $\begin{array}{l}\text { Platelets } \\
\left(10^{9} / \mathrm{L}\right)\end{array}$ & $\begin{array}{c}297.0 \\
(259.0-327.0)\end{array}$ & $\begin{array}{c}304.0 \\
(274.8-337.3)\end{array}$ & $\begin{array}{c}253.0 \\
(242.0-288.0)\end{array}$ & 0.06 & $\begin{array}{c}294.0 \\
(247.5-359.5)\end{array}$ & $\begin{array}{c}264.5 \\
(223.0-314.0)\end{array}$ & $\begin{array}{c}283.0 \\
(235.5-343.5)\end{array}$ & 0.299 \\
\hline MPV (fL) & $\begin{array}{c}6.2 \\
(5.5-6.8)\end{array}$ & $\begin{array}{c}6.1 \\
(5.5-6.6)\end{array}$ & $\begin{array}{c}6.8 \\
(6.1-7.5)\end{array}$ & 0.06 & $\begin{array}{c}7.2 \\
(6.2-7.8)\end{array}$ & $\begin{array}{c}7.4 \\
(6.4-9.2)\end{array}$ & $\begin{array}{c}7.9 \\
(7.0-10.2)\end{array}$ & 0.675 \\
\hline $\begin{array}{l}\text { Glucose } \\
(\mathrm{mmol} / \mathrm{L})\end{array}$ & $\begin{array}{c}4.6 \\
(4.0-4.7)\end{array}$ & $\begin{array}{c}4.9 \\
(4.4-5.1)\end{array}$ & $\begin{array}{c}4.8 \\
(4.4-5.0)\end{array}$ & 0.08 & $\begin{array}{c}11.6 \\
(10.5-18.7)\end{array}$ & $\begin{array}{c}14.1 \\
(8.9-19.0)\end{array}$ & $\begin{array}{c}11.7 \\
(7.8-16.9)\end{array}$ & 0.502 \\
\hline $\operatorname{RBC}\left(10^{12} / \mathrm{L}\right)$ & $\begin{array}{c}5.2 \\
(4.9-5.3)\end{array}$ & $\begin{array}{c}5.2 \\
(4.9-5.4)\end{array}$ & $\begin{array}{c}5.3 \\
(5.1-5.6)\end{array}$ & 0.136 & $\begin{array}{c}4.8 \\
(4.5-5.1)\end{array}$ & $\begin{array}{c}4.9 \\
(4.6-5.1)\end{array}$ & $\begin{array}{c}4.9 \\
(4.6-5.2)\end{array}$ & 0.491 \\
\hline $\begin{array}{l}\text { Hemoglobin } \\
(\mathrm{g} / \mathrm{L})\end{array}$ & $\begin{array}{c}138.0 \\
(131.0-142.0)\end{array}$ & $\begin{array}{c}143.0 \\
(136.8-147.0)\end{array}$ & $\begin{array}{c}149.0 \\
(146.0-156.0)\end{array}$ & $<0.001$ & $\begin{array}{c}129.0 \\
(126.5-139.5)\end{array}$ & $\begin{array}{c}136.0 \\
(129.8-144.0)\end{array}$ & $\begin{array}{c}141.0 \\
(135.5-143.5)\end{array}$ & 0.05 \\
\hline $\begin{array}{l}\text { Hematocrit } \\
(\%)\end{array}$ & $\begin{array}{c}41.2 \\
(39.7-42.8)\end{array}$ & $\begin{array}{c}42.9 \\
(41.3-44.5)\end{array}$ & $\begin{array}{c}45.1 \\
(43.4-46.8)\end{array}$ & $<0.001$ & $\begin{array}{c}39.2 \\
(36.9-39.6)\end{array}$ & $\begin{array}{c}40.6 \\
(38.9-43.1)\end{array}$ & $\begin{array}{c}43.0 \\
(40.6-46.7)\end{array}$ & $<0.001$ \\
\hline MCV (fL) & $\begin{array}{c}80.9 \\
(80.0-82.2)\end{array}$ & $\begin{array}{c}82.9 \\
(81.2-85.5)\end{array}$ & $\begin{array}{c}85.7 \\
(82.9-88.4)\end{array}$ & $<0.001$ & $\begin{array}{c}79.2 \\
(77.1-81.5)\end{array}$ & $\begin{array}{c}82.4(80.4- \\
86.5)\end{array}$ & $\begin{array}{c}88.4 \\
(85.3-92.4)\end{array}$ & $<0.001$ \\
\hline $\mathrm{MCH}(p g)$ & $\begin{array}{c}27.0 \\
(26.4-27.7)\end{array}$ & $\begin{array}{c}27.5 \\
(26.8-28.4)\end{array}$ & $\begin{array}{c}28.2 \\
(27.4-29.3)\end{array}$ & 0.01 & $\begin{array}{c}27.7 \\
(25.7-29.0)\end{array}$ & $\begin{array}{c}27.9(27.0- \\
28.9)\end{array}$ & $\begin{array}{c}28.3 \\
(27.5-30.1)\end{array}$ & 0.481 \\
\hline $\mathrm{MCHC}(\mathrm{g} / \mathrm{L})$ & $\begin{array}{c}333.0 \\
(327.0-336.0)\end{array}$ & $\begin{array}{c}330.0 \\
(327.0-335.3)\end{array}$ & $\begin{array}{c}332.0 \\
(324.0-338.0)\end{array}$ & 0.592 & $\begin{array}{c}343.0 \\
(327.5-366.5)\end{array}$ & $\begin{array}{c}337.0 \\
(323.8-349.3)\end{array}$ & $\begin{array}{c}320.0 \\
(308.0-335.5)\end{array}$ & 0.020 \\
\hline RDW (\%CV) & $\begin{array}{c}11.8 \\
(11.6-11.9)\end{array}$ & $\begin{array}{c}11.7 \\
(11.4-12.0)\end{array}$ & $\begin{array}{c}11.9 \\
(11.3-12.4)\end{array}$ & 0.467 & $\begin{array}{c}11.1 \\
(10.2-12.1)\end{array}$ & $\begin{array}{c}11.5 \\
(10.3-12.1)\end{array}$ & $\begin{array}{c}11.8 \\
(11.2-15.1)\end{array}$ & 0.007 \\
\hline WBC $\left(10^{12} / L\right)$ & $\begin{array}{c}7.3 \\
(5.3-9.6)\end{array}$ & $\begin{array}{c}7.2 \\
(5.9-8.4)\end{array}$ & $\begin{array}{c}7.5 \\
(6.4-8.7)\end{array}$ & 0.776 & $\begin{array}{c}7.1 \\
(5.9-8.7)\end{array}$ & $\begin{array}{c}5.9 \\
(4.8-7.4)\end{array}$ & $\begin{array}{c}5.9 \\
(5.2-8.1)\end{array}$ & 0.126 \\
\hline
\end{tabular}

Values represent medians (lower-upper quartile), $\mathrm{p}^{*}$ - significance between age groups in healthy controls. $\mathrm{p}^{* *}-$ significance between age groups in children with type 1 diabetes. All differences were tested using the Kruskal-Wallis test.

$\mathrm{BMI}$, body mass index; $\mathrm{HbA1c}$, hemoglobin A1c; MPV, mean platelet volume; RBC, red blood cells; MCV, mean corpuscular volume; $\mathrm{MCH}$, mean corpuscular hemoglobin; MCHC, mean corpuscular hemoglobin concentration; RDW, red blood cell distribution width; WBC, white blood cells.

what worse level of disease control, while $30 \%$ of children with T1DM had essentially uncontrolled disease with $\mathrm{HbA} 1 \mathrm{c}$ levels reaching $15 \%$ or more (Table IV).

No significant differences were observed at the level of age, BMI, WBC, hemoglobin, $M C V, M C H$, MCHC, RDW, PLT count, and MPV between compared groups (Table V). Statistically significant differences between groups were observed only for erythrocyte count and hematocrit value $(p=0.013$ and 0.019 , respectively), in addition to expected differences in glucose and $\mathrm{HbA} 1 \mathrm{c}$ levels (Table V).
Spearman correlation analysis (Table VI) showed significant positive correlations between BMI, HGB, $\mathrm{HCT}, \mathrm{MCV}, \mathrm{RDW}$, and age in diabetic Bosnian children. MCHC showed a significant negative correlation with age. MPV showed significant negative correlations with platelet and leukocyte counts, and significant positive correlations with hematocrit and RDW. The number of erythrocytes, as well as white blood cells, showed a significant positive correlation with $\mathrm{HbA} 1 \mathrm{c}$. Other correlations of hematological indices with $\mathrm{HbA} 1 \mathrm{c}$ and glucose in diabetic children 
Table IV Type 1 diabetes mellitus patients grouped by levels of HbA1c.

\begin{tabular}{|l|c|c|c|c|}
\hline & HbA1c $<7 \%$ & HbA1c 7.1-8.4\% & HbA1c $>8.5 \%$ & Total \\
\hline Healthy Controls & 100 & 0 & 0 & 100 \\
\hline Type 1 diabetes & 26 & 44 & 30 & 100 \\
\hline Total & 126 & 44 & 30 & 200 \\
\hline
\end{tabular}

Values represent frequency, number of children in each group determined by values of HbA1c (hemoglobin A1c).

Table $\mathbf{V}$ Influence of glycemia control on the values of anthropometric and hematological parameters in children with type 1 diabetes.

\begin{tabular}{|c|c|c|c|c|}
\hline Parameter & $\mathrm{HbA} 1 \mathrm{c}<7 \%(\mathrm{~N}=26)$ & $\begin{array}{c}\mathrm{HbA} 1 \mathrm{c} 7.1-8.4 \% \\
(\mathrm{~N}=44)\end{array}$ & $\begin{array}{c}\mathrm{HbA} 1 \mathrm{c}>8.5 \% \\
(\mathrm{~N}=30)\end{array}$ & $\mathrm{p}$ \\
\hline Age (years) & $10.0(8.8-12.0)$ & $10.5(8.0-15.0)$ & $11.5(8.8-13.3)$ & 0.886 \\
\hline BMI $\left(\mathrm{kg} / \mathrm{m}^{2}\right)$ & $17.2(15.7-18.9)$ & $18.5(16.4-20.8)$ & $18.4(16.9-20.3)$ & 0.362 \\
\hline HbA1c (\%) & $6.6(6.1-6.9)$ & $7.9(7.4-8.2)$ & $9.3(8.7-10.5)$ & $<0.001$ \\
\hline Platelets $\left(10^{9} / \mathrm{L}\right)$ & $261.0(229.3-307.3)$ & $272.0(234.0-335.0)$ & $288.0(239.8-358.8)$ & 0.181 \\
\hline MPV (fL) & $7.4(6.5-8.9)$ & $7.6(6.7-9.3)$ & $7.5(6.1-9.4)$ & 0.316 \\
\hline Glucose $(\mathrm{mmol} / \mathrm{L})$ & $11.2(6.1-14.5)$ & $12.7(9.7-16.6)$ & $18.6(11.5-21.5)$ & $<0.001$ \\
\hline $\operatorname{RBC}\left(10^{12} / \mathrm{L}\right)$ & $4.6(4.4-5.1)$ & $4.9(4.7-5.1)$ & $4.9(4.7-5.3)$ & 0.013 \\
\hline Hemoglobin $(\mathrm{g} / \mathrm{L})$ & $134.0(125.8-144.0)$ & $140.0(131.0-143.0)$ & $137.0(131.5-144.0)$ & 0.276 \\
\hline Hematocrit (\%) & $40.1(37.8-41.3)$ & $41.0(39.5-44.3)$ & $41.3(39.0-44.4)$ & 0.019 \\
\hline$M C V(f L)$ & $83.9(81.0-87.5)$ & 84.7 (79.9-89.5) & $81.8(80.1-87.4)$ & 0.730 \\
\hline $\mathrm{MCH}(\mathrm{pg})$ & $28.7(27.5-29.8)$ & $28.1(26.8-28.9)$ & $27.7(26.9-29.2)$ & 0.114 \\
\hline $\mathrm{MCHC}(\mathrm{g} / \mathrm{L})$ & $343.0(329.0-358.0)$ & $328.0(315.3-340.5)$ & 333.5 (319.8-346.3) & 0.183 \\
\hline RDW (\%CV) & $11.6(10.5-12.1)$ & $11.6(10.8-12.9)$ & $11.4(10.5-12.6)$ & 0.752 \\
\hline WBC $\left(10^{12} / L\right)$ & $5.6(4.8-6.9)$ & $5.9(4.9-7.9)$ & $6.9(5.7-7.9)$ & 0.067 \\
\hline
\end{tabular}

Values represent medians (lower-upper quartile), p - significance between HbA1c level groups in diabetic children. All differences were tested using the Kruskal-Wallis test.

BMI, body mass index; HbA1c, hemoglobin A1c; MPV, mean platelet volume; RBC, red blood cells; MCV, mean corpuscular volume; $\mathrm{MCH}$, mean corpuscular hemoglobin; $\mathrm{MCHC}$, mean corpuscular hemoglobin concentration; RDW, red blood cell distribution width; WBC, white blood cells.

were not significant. Spearman correlation between erythrocyte and $\mathrm{HbA} 1 \mathrm{c}$ values showed a significant positive correlation (Spearman rho $=0.208$ with $\mathrm{p}=0.037$ ). Spearman correlation between white blood cells and $\mathrm{HbA} 1 \mathrm{c}$ values showed a significant negative correlation (Spearman $\mathrm{rho}=0.222$ with $\mathrm{p}=0.027$ ).

Spearman correlation in healthy Bosnian children (Table VII) also showed significant positive correlations between BMI, HGB, HCT, MCV, and age.
$\mathrm{MCH}$ and erythrocytes showed positive correlations with age as well. PLT showed a significant negative correlation with age in healthy children. HbA1c showed significant negative correlations with $\mathrm{MCH}$ and $\mathrm{MCHC}$, and a significant positive correlation with RDW. Other correlations of hematological indices with $\mathrm{HbA} 1 \mathrm{c}$ and glucose, in healthy children, were not significant. The number of erythrocytes and white blood cells did not show a significant correlation with HbA1c. 
Table VI Spearman correlation for analysed parameters in children with type 1 diabetes mellitus $(N=100)$.

\begin{tabular}{|c|c|c|c|c|c|c|c|c|c|c|c|c|c|c|}
\hline & & AGE & BMI & $\mathrm{HbA1c}$ & PLT & MPV & GLU & RBC & $\mathrm{HGB}$ & HCT & MCV & $\mathrm{MCH}$ & $\mathrm{MCHC}$ & RDW \\
\hline \multirow{2}{*}{ BMI } & $\begin{array}{l}\text { Spearman } \\
\rho \text { (rho) }\end{array}$ & $.496^{* *}$ & / & / & / & / & I & / & / & / & I & / & I & / \\
\hline & $\mathrm{p}$ & $<0.001$ & & & & & & & & & & & & \\
\hline \multirow{2}{*}{$\mathrm{HbA} 1 \mathrm{c}$} & $\begin{array}{c}\text { Spearman } \\
\rho \text { (rho) }\end{array}$ & 0.009 & 0.117 & / & / & / & I & / & / & / & / & / & I & / \\
\hline & $p$ & 0.926 & 0.245 & & & & & & & & & & & \\
\hline \multirow{2}{*}{ PLT } & $\begin{array}{c}\text { Spearman } \\
\rho \text { (rho) }\end{array}$ & 0.012 & 0.188 & 0.173 & I & / & I & / & I & / & I & I & I & I \\
\hline & $\mathrm{p}$ & 0.909 & 0.062 & 0.086 & & & & & & & & & & \\
\hline \multirow{2}{*}{ MPV } & $\begin{array}{l}\text { Spearman } \\
\rho \text { (rho) }\end{array}$ & 0.185 & 0.122 & -0.073 & $-0.33 * *$ & / & I & / & / & / & I & / & I & / \\
\hline & $p$ & 0.065 & 0.225 & 0.470 & 0.001 & & & & & & & & & \\
\hline \multirow{2}{*}{ GLU } & $\begin{array}{c}\text { Spearman } \\
\rho \text { (rho) }\end{array}$ & -0.053 & -0.054 & $0.416^{* *}$ & -0.007 & 0.029 & / & / & / & / & / & / & / & / \\
\hline & $\mathrm{p}$ & 0.597 & 0.591 & $<00.001$ & 0.942 & 0.774 & & & & & & & & \\
\hline \multirow{2}{*}{$\mathrm{RBC}$} & $\begin{array}{c}\text { Spearman } \\
\rho \text { (rho) }\end{array}$ & 0.053 & 0.082 & $0.208^{*}$ & 0.015 & 0.004 & -0.040 & / & I & / & I & / & I & / \\
\hline & $\mathrm{p}$ & 0.597 & 0.420 & 0.037 & 0.883 & 0.969 & 0.694 & & & & & & & \\
\hline \multirow{2}{*}{ HGB } & $\begin{array}{c}\text { Spearman } \\
\rho \text { (rho) }\end{array}$ & $0.268 * *$ & 0.26 ** & 0.049 & 0.052 & 0.058 & -0.104 & $0.706^{* *}$ & / & / & I & I & / & I \\
\hline & $\mathrm{p}$ & 0.007 & 0.008 & 0.625 & 0.605 & 0.567 & 0.305 & $<00.001$ & & & & & & \\
\hline \multirow{2}{*}{$\mathrm{HCT}$} & $\begin{array}{l}\text { Spearman } \\
\rho \text { (rho) }\end{array}$ & $0.401 * *$ & $0.28^{* *}$ & 0.132 & 0.035 & $0.199 *$ & -0.046 & $0.673^{* *}$ & $0.690 * *$ & / & / & / & / & / \\
\hline & $\mathrm{p}$ & $<00.001$ & 0.004 & 0.191 & 0.728 & 0.047 & 0.653 & $<00.001$ & $<00.001$ & & & & & \\
\hline \multirow{2}{*}{ MCV } & $\begin{array}{c}\text { Spearman } \\
\rho \text { (rho) }\end{array}$ & $0.407^{* *}$ & $0.255^{*}$ & -0.101 & 0.096 & 0.178 & -0.041 & $-0.310 * *$ & 0.081 & $0.431 * *$ & I & I & / & / \\
\hline & $\mathrm{p}$ & $<00.001$ & 0.011 & 0.315 & 0.342 & 0.077 & 0.687 & 0.002 & 0.421 & $<00.001$ & & & & \\
\hline \multirow{2}{*}{$\mathrm{MCH}$} & $\begin{array}{c}\text { Spearman } \\
\rho \text { (rho) }\end{array}$ & 0.178 & 0.183 & -0.145 & 0.065 & 0.072 & -0.059 & $-0.455^{* *}$ & $0.207^{*}$ & -0.039 & $0.511 * *$ & I & / & / \\
\hline & $\mathrm{p}$ & 0.077 & 0.069 & 0.150 & 0.520 & 0.476 & 0.559 & $<00.001$ & 0.039 & 0.699 & $<00.001$ & & & \\
\hline \multirow{2}{*}{$\mathrm{MCHC}$} & $\begin{array}{l}\text { Spearman } \\
\rho \text { (rho) }\end{array}$ & $-0.277^{* *}$ & -0.151 & -0.094 & -0.047 & -0.176 & -0.021 & -0.146 & 0.152 & $-0.529 * *$ & $-0.477 * *$ & $0.418 * *$ & / & / \\
\hline & $\mathrm{p}$ & 0.005 & 0.134 & 0.354 & 0.640 & 0.079 & 0.836 & 0.148 & 0.132 & $<00.001$ & $<00.001$ & $<00.001$ & & \\
\hline \multirow{2}{*}{ RDW } & $\begin{array}{l}\text { Spearman } \\
\rho \text { (rho) }\end{array}$ & $0.242^{*}$ & $0.228^{*}$ & -0.012 & 0.128 & $0.499 * *$ & -0.023 & 0.103 & 0.113 & 0.124 & 0.046 & 0.019 & -0.089 & / \\
\hline & $\mathrm{p}$ & 0.015 & 0.022 & 0.907 & 0.203 & $<00.001$ & 0.824 & 0.307 & 0.263 & 0.221 & 0.647 & 0.850 & 0.381 & \\
\hline \multirow{2}{*}{ WBC } & $\begin{array}{c}\text { Spearman } \\
\rho \text { (rho) }\end{array}$ & -0.114 & 0.061 & $0.222 *$ & $0.560 * *$ & $-0.277^{* *}$ & 0.109 & 0.017 & 0.011 & 0.059 & 0.114 & 0.074 & -0.042 & -0.090 \\
\hline & $p$ & 0.259 & 0.548 & 0.027 & $<00.001$ & 0.005 & 0.280 & 0.870 & 0.911 & 0.561 & 0.260 & 0.463 & 0.681 & 0.371 \\
\hline
\end{tabular}

All correlations were tested using Spearman test. Spearman $\rho$ (rho), value of Spearman's rank correlation coefficient, where »-« represents negative correlation, ${ }^{* *}$ " correlation is significant at the 0.01 level, »* « correlation is significant at the 0.05 level. p - significance of correlation between parameters.

$\mathrm{BMI}\left(\mathrm{kg} / \mathrm{m}^{2}\right)$, body mass index; HbA1c $(\%)$, hemoglobin A1c; PLT $\left(10^{9} / \mathrm{L}\right)$, platelets; MPV (fL), mean platelet volume; GLU (mmol/L), glucose; RBC (10 $12 / \mathrm{L})$, red blood cells; HGB (g/L), hemoglobin; HCT (\%), hematocrit; MCV (fL), mean corpuscular volume; $M C H$ (pg), mean corpuscular hemoglobin; $\mathrm{MCHC}(\mathrm{g} / \mathrm{L})$, mean corpuscular hemoglobin concentration; RDW (\%CV), red blood cell distribution width; WBC $\left(10^{12} / L\right)$, white blood cells. 
Table VII Spearman correlation for analysed parameters in healthy children $(\mathrm{N}=100)$.

\begin{tabular}{|c|c|c|c|c|c|c|c|c|c|c|c|c|c|c|}
\hline & AGE & $\mathrm{BMI}$ & $\mathrm{HbA} 1 \mathrm{c}$ & PLT & MPV & GLU & $\mathrm{RBC}$ & HGB & HCT & MCV & $\mathrm{MCH}$ & $\mathrm{MCHC}$ & RDW & \\
\hline \multirow[t]{2}{*}{$\mathrm{BMI}$} & $\begin{array}{c}\text { Spearman } \\
\rho \text { (rho) }\end{array}$ & $.582 * *$ & I & / & / & / & / & / & / & / & / & 1 & / & I \\
\hline & $\mathrm{p}$ & .000 & & & & & & & & & & & & \\
\hline \multirow[t]{2}{*}{$\mathrm{HbA1c}$} & $\begin{array}{c}\text { Spearman } \\
\rho \text { (rho) }\end{array}$ & .009 & -.033 & / & / & / & / & / & / & / & / & / & / & / \\
\hline & $\mathrm{p}$ & .932 & .747 & & & & & & & & & & & \\
\hline \multirow[t]{2}{*}{ PLT } & $\begin{array}{c}\text { Spearman } \\
\rho \text { (rho) }\end{array}$ & $-.314^{* *}$ & -.066 & .134 & / & I & / & / & / & / & / & / & / & / \\
\hline & $\mathrm{p}$ & .001 & .516 & .182 & & & & & & & & & & \\
\hline \multirow[t]{2}{*}{ MPV } & $\begin{array}{c}\text { Spearman } \\
\rho \text { (rho) }\end{array}$ & .191 & $.220^{*}$ & .137 & $-.35 * *$ & / & / & / & / & / & / & / & / & / \\
\hline & p & .057 & .028 & .173 & .000 & & & & & & & & & \\
\hline \multirow[t]{2}{*}{ GLU } & $\begin{array}{c}\text { Spearman } \\
\rho \text { (rho) }\end{array}$ & .103 & -.051 & .168 & -.033 & .040 & / & / & / & / & / & / & / & / \\
\hline & $\mathrm{p}$ & .307 & .615 & .095 & .745 & .695 & & & & & & & & \\
\hline \multirow[t]{2}{*}{$\mathrm{RBC}$} & $\begin{array}{c}\text { Spearman } \\
\rho \text { (rho) }\end{array}$ & $.220^{*}$ & .178 & .050 & .186 & -.068 & -.112 & / & / & / & / & / & / & / \\
\hline & $\mathrm{p}$ & .028 & .076 & .622 & .064 & .500 & .268 & & & & & & & \\
\hline \multirow[t]{2}{*}{$\mathrm{HGB}$} & $\begin{array}{c}\text { Spearman } \\
\rho \text { (rho) }\end{array}$ & $.526^{* *}$ & $.36 * *$ & -.168 & -.015 & -.008 & -.033 & $.660 * *$ & / & / & / & / & I & / \\
\hline & $\mathrm{p}$ & .000 & .000 & .094 & .880 & .936 & .742 & .000 & & & & & & \\
\hline \multirow[t]{2}{*}{ HCT } & \begin{tabular}{|c} 
Spearman \\
$\rho$ (rho)
\end{tabular} & $.538 * *$ & $.42 * *$ & -.029 & .045 & -.004 & -.050 & $.740 * *$ & $.919 * *$ & / & / & I & I & / \\
\hline & $\mathrm{p}$ & .000 & .000 & .775 & .660 & .968 & .622 & .000 & .000 & & & & & \\
\hline \multirow[t]{2}{*}{ MCV } & $\begin{array}{c}\text { Spearman } \\
\rho \text { (rho) }\end{array}$ & $.496 * *$ & $.34 * *$ & -.067 & $-.203^{*}$ & .108 & .021 & $-.317 * *$ & $.348^{* *}$ & $.339 * *$ & / & / & / & / \\
\hline & $\mathrm{p}$ & .000 & .001 & .505 & .043 & .285 & .836 & .001 & .000 & .001 & & & & \\
\hline \multirow[t]{2}{*}{$\mathrm{MCH}$} & \begin{tabular}{|c}
$\begin{array}{c}\text { Spearman } \\
\rho \text { (rho) }\end{array}$ \\
\end{tabular} & $.393 * *$ & $.218^{*}$ & $-.230 *$ & $-.218^{*}$ & .112 & .024 & $-.403 * *$ & $.337^{* *}$ & .165 & $.873^{* *}$ & / & / & / \\
\hline & $\mathrm{p}$ & .000 & .029 & .021 & .029 & .266 & .816 & .000 & .001 & .101 & .000 & & & \\
\hline \multirow[t]{2}{*}{$\mathrm{MCHC}$} & \begin{tabular}{|c} 
Spearman \\
$\rho$ (rho)
\end{tabular} & .049 & -.133 & $-.401 * *$ & -.154 & -.030 & .055 & $-.263^{* *}$ & .112 & $-.234^{*}$ & .085 & $.499 * *$ & / & / \\
\hline & $\mathrm{p}$ & .629 & .189 & .000 & .127 & .767 & .584 & .008 & .269 & .019 & .402 & .000 & & \\
\hline \multirow[t]{2}{*}{ RDW } & $\begin{array}{c}\text { Spearman } \\
\rho \text { (rho) }\end{array}$ & -.006 & .039 & $.304^{* *}$ & .002 & .165 & .007 & .167 & -.170 & .000 & -.173 & $-.356 * *$ & $-.497 * *$ & / \\
\hline & $\mathrm{p}$ & .955 & .697 & .002 & .988 & .101 & .943 & .097 & .091 & .999 & .085 & .000 & .000 & \\
\hline \multirow[t]{2}{*}{ WBC } & \begin{tabular}{|c} 
Spearman \\
$\rho$ (rho)
\end{tabular} & .071 & .103 & -.052 & $.304^{* *}$ & -.132 & -.052 & .108 & -.004 & .016 & -.085 & -.088 & .021 & .007 \\
\hline & $\mathrm{p}$ & .480 & .308 & .607 & .002 & .191 & .604 & .286 & .966 & .876 & .398 & .383 & .835 & .944 \\
\hline
\end{tabular}

All correlations were tested using the Spearman test. Spearman (rho), the value of Spearman's rank correlation coefficient, where »-« represents negative correlation, »** « correlation is significant at the 0.01 level, »* « correlation is significant at the 0.05 level. $\mathrm{p}$ - the significance of correlation between parameters. BMI $\left(\mathrm{kg} / \mathrm{m}^{2}\right)$, body mass index; HbA1c (\%), hemoglobin A1c; PLT (109/L), platelets; MPV (fL), mean platelet volume; GLU (mmol/L), glucose; RBC (10 12/L), red blood cells; HGB (g/L), hemoglobin; HCT (\%), hematocrit; $\mathrm{MCV}(\mathrm{fL})$, mean corpuscular volume; $\mathrm{MCH}(\mathrm{pg})$, mean corpuscular hemoglobin; $\mathrm{MCHC}(\mathrm{g} / \mathrm{L})$, mean corpuscular hemoglobin concentration; RDW (\%CV), red blood cell distribution width; WBC $\left(10^{12} / \mathrm{L}\right)$, white blood cells.

\section{Discussion}

Altered levels of many hematological parameters (red blood cells, white blood cells, platelet function) and their corresponding indices (HGB, HCT, $M C V, M P V)$ have been observed in patients with dia- betes. However, some authors did not report such differences except for higher WBC count in diabetics. In this study, hematological parameters were tested and compared in a group of children with diabetes and their healthy peers. A careful look at our results shows many changes in the diabetic population, one of 
them also being white blood cell count which was lower in children with type 1 diabetes mellitus, mean value $6.49 \times 10^{12}$, compared to their healthy peers (mean $7.44 \times 10^{12}$ leukocytes, $\left.\mathrm{p}<0.001\right)$.

Observed differences were neither sex nor age affected. Similar results related to the lower number of leukocytes in pathogenesis of type 1 diabetes were reported by Harsunen et al. (16). The discrepancy in reported leukocyte counts observed in different studies might be explained by their measurements in different stages of diabetes or from the fact that the data are related to different ethnic groups. Therefore, further investigations are required to explain these differences in WBC counts.

From all white blood cells, neutrophils are most involved in the pathogenesis of type 1 diabetes; thus, WBC count alters mainly due to alteration in neutrophil count. Early studies showed that type 1 diabetes patients had higher white blood cells, primarily neutrophil numbers than the controls (17), and increased neutrophil counts were reported to correlate with an augmented cardiovascular complication risk (17). However, more recent studies showed that circulating neutrophil number and white blood cells consequently were decreased in patients with type 1 diabetes and healthy autoantibody positive subjects, which might be associated with cell specific autoimmunity (18-21). Lower Neutrophil count mediates a decrease in total white blood cell count.

Although our research did not include neutrophil counts, WBC data correlates with the second, more recent studies of Valle et al. (18), Harsunen et al. (19), Wang et al. (20), and Battaglia (21). We found significantly lower WBC values in children with T1DM, compared to healthy controls.

When other hematology data are concerned, descriptive statistics analysis in our study shows a lower number of erythrocytes, lower hematocrit, and hemoglobin levels in patients with type 1 diabetes mellitus when compared to their healthy peers. The Mann-Whitney $U$ test shows a significant difference between healthy group and children with T1DM in number of erythrocytes, hematocrit and hemoglobin values, with $\mathrm{p}<0.001$ for all three parameters analyzed. Interestingly, the number of erythrocytes and the level of hemoglobin were significantly lower in the diabetic female population, whereas MPV was significantly higher when compared to the male diabetic population. Gender differences were not apparent in the healthy population. Hemoglobin, hematocrit, and MCV values also differ by age in both diabetic and healthy children. Mean hemoglobin values in children with type 1 diabetes mellitus in our research were lower when compared to healthy peers, 137.2 vs. $143.4 \mathrm{~g} / \mathrm{L}$, respectively. This is in accordance with results reported by Khudhur et al. (22), Mbata et al. (23), and Kothari et al. (24), who reported lower mean values for RBC, HGB, and HCT in diabetics when compared to controls. All of the abovementioned parameters are indicators for the future development of anemia, a common finding in patients with diabetes, particularly in those with nephropathy and renal impairment as well as retinopathy and macrovascular disease.

When other anemia associated hematological indexes were examined such as $\mathrm{MCH}$ and $\mathrm{MCHC}$, differences at the level of different populations as well as gender differences were not evident. However, age differences in values of MCHC and RDW for diabetic, and $\mathrm{MCH}$ for healthy children, were evident.

One of the red blood cells indicators often investigated for its association with anemia in diabetic patients is red blood cell distribution with (RDW). Mean RDW values in our study did not differ significantly between healthy and children with type 1 diabetes, 11.81 vs. $11.96 \%$, respectively, with $p=0.088$ determined by the Man-Whitney. Similar results were reported in numerous other studies $(29,30)$. Contrary to these data, Biadgo et al. (10), in a similar comparative study on 296 participants, reported a significant difference in red blood cell distribution width between patients with diabetes and healthy controls. Differences across the study populations, as well as differences in study design and ethnicity, may account for the variability of RDW across studies.

Knowing that diabetes is considered to be a prothrombotic condition where platelets play an active role (33-35), it was of great interest to examine the differences in platelet size and number between diabetic and healthy populations. In this context, we were especially interested in MPV since this parameter represents a direct marker of platelet function and activity (9). Several studies have reported higher MPV values in individuals with both T1DM and T2DM, and these changes have been linked to metabolic control of the disease $(31,32)$. The average MPV values for healthy Bosnian children were $6.32 \mathrm{fL}$, while their counterparts with type one diabetes had an average MPV value at $8.32 \mathrm{fL}$. This difference, as determined by the Mann-Whitney $U$ test, was significant with $p<0.001$. Similar results as ours were reported in diabetic children by Venkatesh et al. (36), Demirtunc et al. (37), Zuberi et al. (38), Biaggo et al. (10), Winocour et al. (39), and Çoban et al. $(40,41)$.

Interestingly, Uko et al. (14) observed a significant positive correlation between high PLT and WBC count and raised blood sugar level among the diabetic subjects studied $(r=0.52$ and 0.45$)$, respectively. Other studies showed positive correlations between WBC and $\mathrm{HbA} 1 \mathrm{c}$, such as a recent meta-analysis of 20 studies, which included 90,000 participants where a positive correlation between increased WBC level and diabetes risk was found (45).

In Sulochana et al. (46) study among the 100 type 2 diabetic patients with increased glycated hemoglobin, total leukocyte count was elevated in 
about 18 of 46 male and 22 of 54 female patients. In Bosnian children with type 1 diabetes, WBC count showed a significant negative correlation with $\mathrm{HbA} 1 \mathrm{c}$ and glucose. In the study by Milosevic et al. (11), no such correlation was reported on patients with type 2 diabetes. Reported results are contradictory and require further attention.

The effects of glucose control and levels of $\mathrm{HbA1c}$ (indicators of disease control) on hematological parameters were investigated in this study in three groups of children.

We expected significant alterations in RDW and other RBC parameters reported in other studies (47). In our study, statistically significant differences between groups with different levels of disease control were observed only for erythrocyte count and hematocrit value ( $p=0.013$ and 0.019 , respectively), excluding expected differences in glucose and $\mathrm{HbA} 1 \mathrm{c}$ levels. When assessing the correlation between $\mathrm{HbA} 1 \mathrm{c}$ and hematological indices in diabetic children and their healthy peers, in Bosnian children, the number of erythrocytes, as well as white blood cells, showed a significant positive correlation with $\mathrm{HbA} 1 \mathrm{c}$ and at the same time negative correlation with $\mathrm{MCH}$ and $\mathrm{MCHC}$ and positive for RDW in healthy children. Interestingly, some (49) authors reported a significant positive correlation between MPV and $\mathrm{HbA} 1 \mathrm{c}$ $(R=306, p<0.005)$, while others on non-diabetic subjects observed an inverse correlation between $\mathrm{HbA} 1 \mathrm{c}$ and MCV $(r=-0.22, \mathrm{p}<0.05), \mathrm{MCH}(r=-$ $0.30, p<0.05)$, and MCHC $(r=-0.32, p<0.05)$ (51). MCV, MCH, and MCHC showed no significant correlation with $\mathrm{HbA} 1 \mathrm{c}$ in numerous studies reported $(47,50)$. On the contrary, Jaman et al. (48) reported a statistically significant decrease of HCT in diabetic patients (HbA1c 7.5) when compared with non-diabetic patients (HbA1c 6.5). A decrease in the HCT value of diabetic patients may be due to the dehydration and accumulation of protein.

Ulutaş et al. (42) also reported a positive correlation between MPV level and $\mathrm{HbA} 1 \mathrm{c}$ level, which is following our results. Hekimsoy et al. (43), have shown the opposite, while Ersoy et al. (44), reported similar MPV values in children diagnosed with T1DM to those in a control group (8.74 \pm 0.96 and $8.49 \pm$ 0.66 , respectively). These different results in platelet count could be explained by their dependency on several variables, such as mean platelet survival, platelet production rate, and turnover rate in diabetes mellitus. Our study has some limitations related to the effects of the long-term course of the disease, and these limitations have to be overcome in future studies.

\section{Conclusions}

In this study, statistically significant differences in numerous hematological parameters of diabetic Bosnian children were observed when compared to healthy controls. The mean values of erythrocytes, hematocrit, and hemoglobin for diabetic children were found to be within reference ranges but significantly lower than the values of the control group, indicating possible development of anemia in the former group. At the same time, MCV, MCH, and MPV were higher in children with Type 1 diabetes mellitus indicating an alteration in erythrocyte morphology. Hemoglobin, hematocrit, MCV, MCHC, and RDW were age-dependent. Erythrocyte count, hemoglobin, and MCV showed significant gender differences. Age and gender had no effect on MPV, WBC, glucose, or $\mathrm{HbA} 1 \mathrm{c}$ levels in children with type 1 diabetes mellitus. The mean value of white blood cells was also found to be lower in diabetic children, supporting a general involvement of the innate immune system in the pathogenesis of type 1 diabetes. Significant differences in mean values of erythrocyte and white blood cells were supported by observed correlations between $\mathrm{HbA} 1 \mathrm{c}$ and: WBC and RBC. A significant positive correlation between $\mathrm{WBC}$ and $\mathrm{HbA} 1 \mathrm{c}$, as well as RBC and $\mathrm{HbA} 1 \mathrm{c}$, was observed.

Our findings suggest that hematological indices as accessible, simple, inexpensive laboratory parameters could be a useful tool in following up T1DM in children. MPV, in addition to $\mathrm{HbA} 1 \mathrm{c}$, can serve as a prognostic tool for determining the risk of diabetic microvascular and macrovascular complications.

Acknowledgments. This study was supported by a grant received from the Federal Ministry of Science and Education for the 2017 year awarded to MM. Project title: The importance of determining the parameters of oxidative stress, inflammation, and hemostasis in the early diagnosis of obesity in the pediatric population, number: 05-39-2571-1/17.

\section{Conflict of interest statement}

The authors stated that they have no conflicts of interest regarding the publication of this article. 


\section{References}

1. Karvonen M, Viik-Kajander M, Moltchanova $E$, et al Incidence of childhood type 1 diabetes worldwide. Diabetes Mondiale (DiaMond) Project Group. Diabetes Care 2000; 23(10): 1516-26.

2. Korkmaz O. Assessment of the Platelet Parameters in Children With Type 1 Diabetes Mellitus. J Endocrinol Metab 2018; 8(6): 144-8.

3. Lipton RB, Drum ML, Danielson KK, et al. Onset features and subsequent clinical evolution of childhood diabetes over several years. Pediatr Diabetes 2011; 12(4 Pt 1): 326.

4. Ersoy M, Elevli M, Ersoy O, et al. Intima-Media Thickness and Mean Platelet Volume in Children With Type 1 Diabetes Mellitus. Iran J Pediatr 2015 April. 25(2): e368.

5. Nathan DM. Long-term complications of diabetes mellitus. N Engl J Med 1993; 328(23).

6. Krishnan S, Short KR. Prevalence and significance of cardiometabolic risk factors in children with type 1 diabetes. J Cardiometab Syndr 2009; 4 (1): 50-6.

7. Malachowska B, Tomasik B, Szadkowska A, et al. Altered platelets' morphological parameters in children with type 1 diabetes - a case-control study. BMC Endocr Disord 2015; Apr 3. 15: 17.

8. Kakouros N, Rade JJ, Kourliouros A, Resar JR. Platelet function in patients with diabetes mellitus: from a theoretical to a practical perspective. Int J Endocrinol 2011; 2011: 742719.

9. Shukla KD, Chandra PK, Pawah KA. Study of hematological indices in patients with diabetes mellitus and hypertensive diabetes mellitus. International Journal of Medicine Research, 2016; 1(4): 28-31.

10. Biadgo B, Melku M, Abebe SM, Abebe M. Hematological indices and their correlation with fasting blood glucose level and anthropometric measurements in type 2 diabetes mellitus patients in Gondar, Northwest Ethiopia. Diabetes Metab Syndr Obes 2016; 17(9): 91-9.

11. Milosevic D, Lukic Panin V. Relationship between hematological parameters and glycemic control in type 2 diabetes mellitus. J Med Biochem 2019; 38(2): 164-71.

12. Gkrania-Klotsas E, Ye Z, Cooper AJ, et al. Differential white blood cell count and type 2 diabetes: systematic review and meta-analysis of cross-sectional and prospective studies. PLoS one 2010; 5(10): e13405.

13. Singh $M$, Shin S. Changes in erythrocyte aggregation and deformability in diabetes mellitus. Indian Journal of Experimental Biology 2009; 47(1): 7-15.

14. Uko EK, Isaac IZ, Abdulrahaman Y, Adias TC, Sani Y, Shehu RS, Liman HM, Dalltu MK and Mainasara AS. Some Haematological Parameters in Patients with Type1 Diabetes in Sokoto, North Western Nigeria. J Blood Lymph 2013, 3: 1.

15. American Diabetes Association. Children and Adolescents: Standards of Medical Care in Diabetes-2019. Diabetes Care Jan 2019, 42 (Supplement 1) S148S164.

16. Harsunen $M H$, Puff $R, D^{\prime}$ Orlando $O$, Giannopoulou $E$, Lachmann L, Beyerlein A, von Meyer A, Ziegler A-G.
Reduced Blood Leukocyte and Neutrophil Numbers in the Pathogenesis of Type 1 Diabetes. Horm Metab Res 2013; 45(06): 467-70.

17. Huang J, Xiao $Y, X u A$, et al. Neutrophils in type 1 diabetes. Journal of diabetes investigation vol. 7,5 (2016): 652-63. doi:10.1111/jdi.12469

18. Valle A, Giamporcaro GM, Scavini M, et al. Reduction of circulating neutrophils precedes and accompanies type 1 diabetes. Diabetes 2013; 62: 2072-7.

19. Harsunen $M H$, Puff $R, D^{\prime}$ Orlando $O$, et al. Reduced blood leukocyte and neutrophil numbers in the pathogenesis of type 1 diabetes. Horm Metab Res 2013; 45: 467-70.

20. Wang $Y$, Xiao $Y$, Zhong $L$, et al. Increased neutrophil elastase and proteinase 3 and augmented NETosis are closely associated with betacell autoimmunity in patients with type 1 diabetes. Diabetes 2014; 63: 4239-48.

21. Battaglia M. Neutrophils and type 1 autoimmune diabetes. Curr Opin Hematol 2014; 21: 8-15.

22. Khudhur HK, Al-Ani HM. Hematological Parameters in Children with Type-1 Diabetes. Medical Journal of Babylon, Vo. 16, Issue 3, July-September 2019.

23. Mbata CA, Adegoke A, Nwagu C, et al. Some Haematological Parameters in Diabetic Patients in Port Harcourt Nigeria. AJMS 2015; 3(2): 2348-7186.

24. Kothari R, Bokariya P. A comparative study of haematological parameters in type I diabetes mellitus patients \& healthy young adolescents. Int J Biol Med Res 2012; 3: 2429-32.

25. Thomas MC, Maclsaac RJ, Tsalamandris C, Power D, Jerums G. Unrecognized anemia in patients with diabetes. Diabetes Care 2002; 24: 1164-9.

26. Oyedemi SO, Yakubu MT, Afolayan AJ. Antidiabetic activities of aqueous leaves extract of Leonotis leonurus in streptozotocin induced diabetic rats. J Med Plant Res 2011; 5: 119-25.

27. Thomas S, Rampersad M. Anaemia in diabetes. Acta Diabetol 2004; 41 Suppl 1: S13-7.

28. Kengne AP, Czernichow S, Hamer M, Batty GD, Stamatakis E. Anaemia, haemoglobin level and cause specific mortality in people with and without diabetes. PLoS One 2012; 7: e41875.

29. Cakir L, Aktas G, Enginyurt O, Cakir S. Mean platelet volume increases in type 2 diabetes mellitus independent of HbA1c level. Acta Medica Mediterranea 2014; 30: 425.

30. Dada OA, Uche E, Akinbami A, et al. The relationship between red blood cell distribution width and blood pressure in patients with type 2 diabetes mellitus in Lagos, Nigeria. J Blood Medicine. 2014; 5: 185-9.

31. Korkmaz O. Assessment of the Platelet Parameters in Children With Type 1 Diabetes Mellitus. J Endocrinol Metab 2018; 8(6): 144-8.

32. Sharpe PC, Trinick T. Mean platelet volume in diabetes mellitus. Q J Med 1993; 86(11): 739-42. 
33. Kodiatte TA, Manikyam UK, Rao SB, Jagadish TM, Reddy M, Lingaiah HK, Lakshmaiah V. Mean platelet volume in Type 2 diabetes mellitus. J Lab Physicians 2012; 4(1): 5-9.

34. Zuberi BF, Akhtar N, Afsar S. Comparison of mean platelet volume in patients with diabetes mellitus, impaired fasting glucose and nondiabetic subjects. Singapore Med J 2008; 49(2): 114-6.

35. Ates $\mathrm{O}$, Kiki I, Bilen $\mathrm{H}$, et al. Association of mean platelet volume with the degree of retinopathy in patients with diabetes mellitus. Eur J Gen Med 2009; 6(2): 99-102.

36. Venkatesh V, Kumar R, Varma DK, Bhatia P, Yadav J, Dayal D. Changes in platelet morphology indices in relation to the duration of disease and glycemic control in children with type 1 diabetes mellitus. J Diabetes Complications 2018; 32(9): 833-8.

37. Demirtunc R, Duman D, Basar $M$, Bilgi $M$, Teomete $M$, Garip T. The relationship between glycemic control and platelet activity in type 2 diabetes mellitus. J Diabetes Complications 2009; 23: 89-94.

38. Zuberi BF, Akhtar N, Afsar S. Comparison of mean platelet volume in patients with diabetes mellitus, impaired fasting glucose and non-diabetic subjects. Singapore Med J 2008; 49: 114-6.

39. Winocour PD. Platelets, vascular disease, and diabetes mellitus. Canadian Journal of Physiology and Pharmacology 1994; 72(3): 295-303.

40. Coban E, Kucuktag S, Basyigit S. Platelet activation in subjects with impaired glucose tolerance. Platelets 2007; 18(8): 591-4.

41. Coban E, Bostan F, Ozdogan M. The mean platelet volume in subjects with impaired fasting glucose. Platelets 2006; 17(1): 67-9.

42. Ulutas KT, Dokuyucu R, Sefil F, Yengil E, Sumbul AT, Rizaoglu $\mathrm{H}$, et al. Evaluation of mean platelet volume in patients with type 2 diabetes mellitus and blood glucose regulation: a marker for atherosclerosis? International journal of clinical and experimental medicine 2014; 7(4): 955-61.
43. Hekimsoy Z, Payzinb B, Ornek T, Kandogan G. Mean platelet volume in Type 2 diabetic patients. J Diabetes Complications 2004; 18: 173-6.

44. Ersoy M, Selcuk Duru HN, Elevli M, Ersoy O, Civilibal M. Aortic intima-media thickness and mean platelet volume in children with type 1 diabetes mellitus. Iran J Pediatr 2015; 25(2): e368.

45. Gkrania-Klotsas E, Ye Z, Cooper AJ, et al. Differential white blood cell count and type 2 diabetes: systematic review and meta-analysis of cross-sectional and prospective studies. PLoS ONE 2010;5: e13405.

46. Sulochana S, Viswanath A. Correlation of total leucocyte count and differential leucocyte count in relation to glycated haemoglobin in type 2 diabetes. Int J Health Sci Res 2017; 7(2): 94-7.

47. Bhutto A, Abbasi A, Abro A. Correlation of Hemoglobin A1c with Red Cell Width Distribution and Other Parameters of Red Blood Cells in Type II Diabetes Mellitus. Cureus, August 30, 2019; 11(8): e5533.

48. Jaman MS, Rahman MS, Swarna RR, Mahato J, Miah $M M$, et al. Diabetes and red blood cell parameters. Ann Clin Endocrinol Metabol 2018; 2: 001-009.

49. Kurt H, Demirkiran D. Changing of Hemoglobin A1c Affects Mean Platelet Volume in Type-2 Diabetes Mellitus. Ulutas Med J 2016; 2(1): 27-35.

50. Rafal W. Wojciak, Mojs E, Stanislawska-Kubiak M. The Occurrence of Iron-Deficiency Anemia in Children With Type 1 Diabetes. Journal of Investigative Medicine \& Volume 62, Number 6, August 2014.

51. Hardikar P, Joshi S, Bhat D, et al. Spuriously high prevalence of prediabetes diagnosed by $\mathrm{HbA} 1 \mathrm{c}$ in young Indians partly explained by hematological factors and iron deficiency anemia. Diabetes Care 2012, 35: 797802.

52. Koga M, Morita S, Saito H, Mukai M, Kasayama S: Association of erythrocyte indices with glycated haemoglobin in pre-menopausal women. Diabet Med 2007, 24: 843-7. 